\title{
Unmet Mental Health Needs Associated With Covid Must Be Addressed
}

\author{
Morgan T. Sammons
}

Published online: 2 February 2022

(c) National Register of Health Service Psychologists 2022

Two years have passed since health officials in the province of Hubei, China, notified world health authorities of a novel coronavirus causing significant respiratory illness. Although the scientific community responded with alacrity and a number of effective vaccines and therapeutic drugs have been rapidly developed and brought to market, the death toll continues to rise. In spite of a robust public health response, over 800,000 deaths in the United States and over 5,300,000 worldwide are attributed to the virus with over $271,000,000$ confirmed cases (World Health Organization, 2021).

Psychological morbidity associated with the pandemic continues to worsen. Though figures remain anecdotal, the available evidence points to unprecedented demand for mental health services. In February 2021, the US Centers for Disease Control and Prevention reported that over $40 \%$ of adults in the US had experienced symptoms of anxiety or depression associated with the epidemic. Unmet demand for mental health services, then estimated at over $11 \%$ of adults or approximately 30 million people (Vahratian et al., 2021), has shown a proportional increase. Although it is far more likely that any growth in unmet demand from one year ago would be exponential rather than arithmetic, a crude extrapolation suggests that over $15 \%$ of the adult population of the United States is currently experiencing an unmet demand for mental health services. In raw terms, then, perhaps over 35 million individuals in need are unable to access mental health resources. We do not have firm indices of acuity, nor do we know the level of effort that individuals have expended in seeking mental health services. Thus, it is likely that this estimate is overlarge. Regardless of the exact number, a sizable portion, perhaps even an epidemic portion, of the US population appears to have unsuccessfully sought mental health services. The main thrust of public health initiatives to this point in the pandemic has been, as is only appropriate, focused on addressing the massive infectious disease component of global illness. As health service psychologists we should now begin to advocate for public health efforts that address long covid and all attendant mental health consequences associated with the long-term sequelae of covid.

Data on the neuropsychological sequelae of covid and long covid are sparse. In the current issue of the Journal of Health Service Psychology, Rivas-Vazquez and colleagues (2022) review the available literature on persistent cognitive and emotional symptoms that may follow a mild acute infection. Their review cited findings that over $13 \%$ of patients with severe initial disease had neurological complications such as toxic encephalopathy, stroke, seizure, or hypoxic injury. The long-covid syndrome, marked by fatigue, anxiety, depression, memory loss, and attentional problems, is evidently not dependent on initial severity of the illness. Such symptoms may persist for six months or more in as many as $30 \%$ of patients with mild acute illness long after markers of infection have disappeared.

Krishnan and colleagues (2022) also discuss the persistence of cognitive impairment after brief episodes of acute illness. Neuropsychological symptoms of diminished attention, impaired executive function, and recall, colloquially termed brain fog or post-acute sequelae of SARS-CoV-2 infection (PASC), are protean but appear to describe a common set of chronic emotional and cognitive deficits that remain in a substantial minority of patients. As these authors note, premorbid psychological symptoms appear to be a risk factor, and other shared derangements in sleep, diet, and affect may contribute to the persistence of this cluster of symptoms. A comprehensive neuropsychological evaluation can assist in establishing a baseline against which treatment progress can be measured. Whatever their etiology, postcovid neuropsychological deficits have emerged as a focus for investigation and intervention. It will be essential for medical and mental health providers to articulate standardized assessment protocols along with symptom-specific interventions.

Two articles in the current issue discuss the psychological effects on providers who work with patients suffering from covid. Miu and colleagues (2022) emphasize the importance 
of peer consultation in a digital era where opportunities for informal interprofessional consultation are lacking. As the pandemic continues, providers and patients face unprecedented personal and professional challenges; these challenges highlight the need for practitioners to implement new strategies for effective consultation.

Compassion fatigue remains an unevenly studied phenomenon among mental health providers. While concepts of vicarious traumatization or secondary traumatic stress have achieved currency in the literature over the past three decades and studies of providers have increased substantially during the pandemic, identification of specific risk factors and vulnerabilities remains lacking. Do the innate vicissitudes of intensive care nursing, for example, protect against the development of compassion fatigue, or do they increase vulnerability? Imprecise nomenclature, the relative lack of standardized assessment devices, and divergent study methods make a clear understanding difficult to achieve. PaivaSalisbury and Schwanz (2022) provide an analysis of some of the challenges associated with a better understanding of the concept. Additional standardized investigations may assist in distinguishing between compassion fatigue and other forms of psychological distress and may help produce a common set of preventive and therapeutic interventions.

Grasso (2022) discusses the rise in incidence of interpersonal violence during the pandemic. While violent incidents have increased, calls to violence hotlines have seen a tremendous decrease in volume, possibly attributable to a lack of safe or private spaces when stay-at-home orders are issued. Hospitalizations due to physical maltreatment have increased, but mandated child protective services reporting has declined. The long-term developmental consequences of childhood exposure to interpersonal violence are welldocumented. What strategies should child behavioral experts and mental health planners consider addressing during an almost certain increase in demand for child and adolescent mental health services stemming from pandemic-related familial violence?

This issue of the Journal of Health Service Psychology illustrates the numerous cognitive, emotional, and psychosocial consequences of covid. Management and prevention of acute infection remain the critical public health priority, but as the pandemic unfolds, increasing attention must be paid to longer-term consequences. We are, as previously noted, experiencing a rapid increase in demand for mental health services that the current mental health workforce cannot meet. The psychological and psychosocial consequences of covid will continue to manifest, and it is improbable that these consequences are transient. As the immediate infectious disease threat diminishes, we must proactively devote resources to developing effective screening and intervention strategies of post-acute covid symptoms. The inefficiencies in educating and training psychologists and other mental health providers have long been recognized. Urgent attention by educators and planners to acknowledge and correct these inefficiencies is required if we care to address the long-term consequences of the disease.

\section{References}

Grasso, D. J. (2022). A Trauma-Informed Approach to Assessment, Case Conceptualization, and Treatment Planning for Youth Exposed to Intimate Partner Violence. Journal of Health Service Psychology, 48(1). https://doi.org/10.1007/s42843-021-00053-2

Krishnan, K., Lin, Y., Prewitt, K. M., and Potter, D. A. (2022). Multidisciplinary Approach to Brain Fog and Related Persisting Symptoms Post COVID-19. Journal of Health Service Psychology, 48(1). https://doi.org/10.1007/s42843-022-00056-7

Miu, A. S., Joseph, A., Hakim, E., Cox, E. D., and Greenwald, E. (2022). Peer Consultation: An Enriching Necessity Rather Than a Luxury for Psychologists During and Beyond the Pandemic. Journal of Health Service Psychology, 48(1). https://doi.org/10. 1007/s42843-021-00052-3

Paiva-Salisbury, M. L. \& Schwanz, K. A. (2022). Building Compassion Fatigue Resilience: Awareness, Prevention, and Intervention for Pre-Professionals and Current Practitioners. Journal of Health Service Psychology, 48(1). https://doi.org/10.1007/ s42843-022-00054-9

Rivas-Vazquez, R. A., Rey, G., Quintana, A., and Rivas-Vazquez, A. A. (2022). Assessment and Management of Long COVID. Journal of Health Service Psychology, 48(1). https://doi.org/10.1007/ s42843-022-00055-8

World Health Organization (2021). WHO coronavirus (Covid-19) dashboard. Accessed December 17, 2021. https://covid19.who.int

Vahratian, A., Blumberg, S. J., Terlizzi, E. P., \& Schiller, J. S. (2021). Symptoms of Anxiety or Depressive Disorder and Use of Mental Health Care Among Adults During the COVID-19 Pandemic United States, August 2020-February 2021. MMWR Morb Mortal Wkly Rep;70, 490-494. https://doi.org/10.15585/mmwr.mm701 $3 \mathrm{e} 2$

Morgan T. Sammons, $\mathrm{PhD}, \mathrm{ABPP}$, is the CEO of the National Register of Health Service Psychologists, and the Editor in Chief of the Journal of Health Service Psychology. He is a retired Navy captain and was formerly the U.S. Navy's specialty leader for clinical psychology. 